\title{
Refrigerant Mass Distribution in an R600a Household Refrigerator- Freezer during Cyclic Operation
}

\author{
Wonhee Cho ${ }^{1}$, DongChan Lee ${ }^{2}$, Dong Soo Jang ${ }^{2}$, Yongchan Kim ${ }^{2}$ \\ ${ }^{1}$ Graduate school of Mechanical Engineering, Korea University \\ Anam-ro 145, Seongbuk-gu, Seoul, Republic of Korea \\ stevenc7@korea.ac.kr \\ ${ }^{2}$ Department of Mechanical Engineering, Korea University \\ Anam-ro 145, Seongbuk-gu, Seoul, Republic of Korea \\ ldc1120@korea.ac.kr; nicebb0y@korea.ac.kr; yongckim@korea.ac.kr
}

\section{Extended Abstract}

With increasing concerns on greenhouse gas emissions and regulations on the use of hydrofluorocarbons by the Kyoto Protocol due to high global warming potential, there is growing need to replace R134a that is commonly used in domestic refrigerator-freezers (RFs) [1]. Many studies on alternative refrigerants for household RFs have been conducted over the years, and the consensus is that the use of hydrocarbons could be the solution to the environmental issues [2]. Especially, R600a is considered to have the most potential in substituting R134a owing to its minimal GWP and wide availability [3]. Hydrocarbons, on the other hand, have flammable and explosive properties so that careful attention to leakage and system charge is necessary [4]. In order to mitigate the safety risk involved with overcharged R600a in an RF system, the refrigerant charge distribution needs to be investigated to locate components where design could be optimized for charge reduction. Previous studies have experimentally measured the real-time charge distribution in conventional vapor compression refrigeration systems and found that the most amount of refrigerant was in the high pressure liquid lines during steady state operation [5]. In addition, earlier studies have shown that the largest displacement of refrigerant happens during changes in the operation modes, which occurs periodically in the compressor on-off controlled RFs. However, research on dynamic characteristics of refrigerant distribution in RFs with more complex configurations of refrigeration cycles is still limited.

Therefore, in this study, a transient numerical model for an R600a is developed to simulate the various RF operation modes and to investigate the transient characteristics of refrigerant distribution under cyclic operation. The RF system under investigation was configured in a parallel cycle and consisted of two individual fresh food-compartment and freezercompartment evaporators, of which the flow paths were dictated by the operation modes. The simulation model is validated by comparing the predicted results with the experimental data from the start-up to cyclic operations. The refrigerant charge distribution is also experimentally measured using a quick-valve method and compared with the numerical result under transient condition. Using the developed simulation model, the characteristics of charge distribution are analysed based on the following variables: ambient temperature, compressor RPM, and total refrigerant charge. The result of this study could provide a guidance for improving the component design and reliability of RFs using R600a by identifying maldistribution of refrigerant charge.

\section{References}

[1] Kyoto protocol to the United Nations framework convention on climate change, UNFCCC, 1992.

[2] K. Park, T. Seo, D. Jung, "Performance of alternative refrigerants for residential air-conditioning applications," Appl. Energy, vol. 84, no. 10, pp. 985-991, 2007.

[3] R. Ciconkov, "Refrigerants: There is still no vision for sustainable solutions," Int. J. Refrig., vol. 86, pp. 441-448, 2018.

[4] F. Poggi, H. Macchi-Tejeda, D. Leducq, A. Bontemps, "Refrigerant charge in refrigerating systems and strategies of charge reduction," Int. J. Refrig., vol. 31, pp. 353-370, 2008.

[5] L. Vaitkus, "Low charge transport refrigerator (I). Refrigerant charge and strategies of charge reduction," Mechanika, vol. 17, no. 6, pp. 665-673, 2011. 